\title{
BMJ Open Investigating the effects of 6 months extended duration, in-centre nocturnal versus conventional haemodialysis treatment: a non-randomised, controlled feasibility study
}

\author{
Darren R Churchward, ${ }^{1,2}$ Matthew P M Graham-Brown, ${ }^{1,2}$ Robert Preston, ${ }^{1}$ \\ Warren Pickering, ${ }^{1}$ Gerry $\mathrm{P}$ McCann, ${ }^{3}$ James O Burton ${ }^{1,2}$
}

To cite: Churchward DR, Graham-Brown MPM, Preston $\mathrm{R}$, et al. Investigating the effects of 6 months extended duration, in-centre nocturnal versus conventional haemodialysis treatment: a non-randomised, controlled feasibility study. BMJ Open 2016:6:e012583.

doi:10.1136/bmjopen-2016012583

- Prepublication history for this paper is available online. To view these files please visit the journal online (http://dx.doi.org/10.1136/ bmjopen-2016-012583).

Received 9 May 2016 Revised 28 July 2016 Accepted 12 August 2016

\section{CrossMark}

\footnotetext{
1John Walls Renal Unit, Leicester General Hospital, Leicester, UK

${ }^{2}$ Department of Infection, Immunity and Inflammation, University of Leicester, Leicester, UK

${ }^{3}$ Department of Cardiology, NIHR Leicester

Cardiovascular Biomedical Research Unit, Glenfield Hospital, Leicester, UK
}

Correspondence to Darren R Churchward; dc262@le.ac.uk

\section{ABSTRACT}

Introduction: In-centre nocturnal haemodialysis (INHD) is an underutilised dialysis regimen that can potentially provide patients with better clinical outcomes due to extended treatment times. We have established an INHD programme within our clinical network, fulfilling a previously unmet patient need. This feasibility study aims to gather sufficient data on numerous outcome measures to inform the design of a multicentre randomised controlled trial that will establish the potential benefits of INHD and increase the availability of this service nationally and internationally.

Methods and analysis: This will be a nonrandomised controlled study. Prevalent patients on haemodialysis (HD) will electively change from a conventional in-centre HD regimen of 4 hours thrice weekly to a regimen of extended treatment times (5-8 hours) delivered in-centre overnight thrice weekly. After recruitment of the INHD cohort, a group of patients matched for age, gender and dialysis vintage will be selected from patients remaining on a conventional daytime dialysis programme. Outcome measures will include left ventricular mass as measured by MRI, physical performance measured by the short physical performance battery and physical activity measured by accelerometry. Additionally we will measure quality of life using validated questionnaires, nutritional status by bioimpedance spectroscopy and food diaries, and blood sampling for markers of cardiovascular disease, systemic inflammation. Suitable statistical tests shall be used to analyse the data. We will use omnibus tests to observe changes over the duration of the intervention and between groups. We will also look for associations between outcome measures that may warrant further investigation. These data will be used to inform the power calculation for future studies.

Ethics and dissemination: A favourable opinion was granted by Northampton Research Ethics Committee (15/ EM/0268). It is anticipated that results of this study will be presented at national and international meetings, with reports being published in journals during 2017.

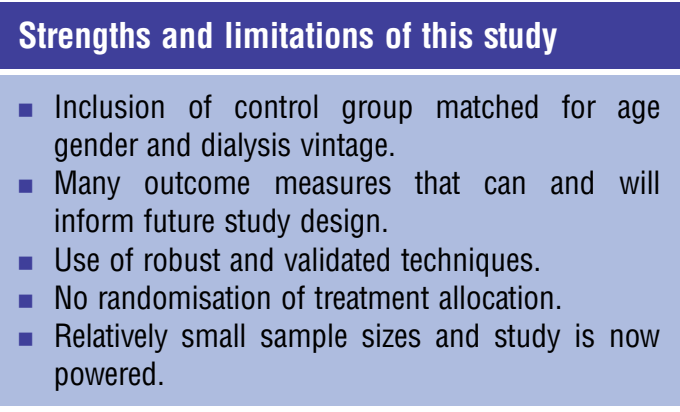

Trial registration number: ISRCTN16672784.

\section{INTRODUCTION}

Incidence and prevalence of chronic kidney disease (CKD) has increased significantly over the last decade. ${ }^{12}$ It is estimated that between $5 \%$ and $8 \%$ of the current UK population have some degree of chronic renal impairment. ${ }^{3}{ }^{4}$ Of these, a small proportion will progress to end-stage renal disease (ESRD) and require some form of renal replacement therapy (RRT). After transplantation, the second most common form of RRT in the UK is haemodialysis $(\mathrm{HD})^{5}$ and according to the latest UK renal registry data ${ }^{5}$ there were 24166 patients on HD in the UK in 2014, an increase of $2 \%$ over 2013. This trend of increasing numbers has been consistent over the last decade.

Patients typically receive HD three times per week for four hours per session. This conventional HD (CHD) regimen has a large physiological impact on a patient's cardiovascular system, ${ }^{6-8}$ and potentially contributes to high rates of cardiovascular disease 
in this population. This is particularly important as cardiovascular death accounts for $40 \%$ of all-cause mortality $^{9}$ in patients on HD. The symptom burden related to HD is also extremely high, ${ }^{10}$ with many patients reporting very poor quality of life. ${ }^{11} 12$ These problems combined with the fact that many patients lose large portions of their time travelling to and from dialysis centres mean that many are unable to continue in paid employment.

The increasing number of patients on CHD is putting increased demand on dialysis services, and slots for HD are now at a premium. Home-based HD is one method of providing extended treatments to patients, but currently only $4.7 \%$ of patients on HD are on a home HD programme. ${ }^{2}$ Although home HD may be more flexible and provide opportunity for more patients to receive treatment, there are significant barriers preventing major usage. These include physician-related factors, such as lack of knowledge and expertise; as well as patient-related factors such as fears and anxieties around self-cannulation or distance from medical care, and perceived caregiver burden. ${ }^{13}$

In-centre nocturnal haemodialysis (INHD) is an alternative method of delivering extended HD to patients that overcomes barriers to home HD for some patients. INHD provides extended periods of HD overnight for prevalent patients on HD in the outpatient setting, and may also help accommodate the increased demand for HD. There are already cross-sectional data that suggest INHD may be associated with improvements in certain clinical outcomes $^{14-19}$ and may even improve survival. ${ }^{20}$ Despite these potential clinical benefits, INHD is not routinely offered as a standard therapy choice in the UK.

The aim of this non-randomised controlled feasibility study is to provide data which will inform future trial design to adequately assess the benefits of INHD. Currently, the majority of trials investigating the effects of extended nocturnal HD involve participants on daily treatments at home. ${ }^{21}$ At the time of publication, evidence for the benefits of INHD mainly suggests improvements in cardiovascular health, specifically with regards to left ventricular hypertrophy (LVH), and blood biochemistry. However, of the papers investigating the effects on LVH, only one has used cardiac MRI (CMR), which is recognised as the gold standard. ${ }^{22}$ No data currently exist on the impact INHD may have on physical performance or physical activity levels.

The MIDNIGHT study (Maintenance In-centre nocturnal haemoDialysis: a feasibility study iNvestIGating the effects on cardiac structure and pHysical funcTion) is the first stage in a programme of planned studies seeking to understand the extent to which longer HD sessions can improve physical and psychological health, as well as how overnight HD programmes can be implemented into routine practice in the UK. The MIDNIGHT study will investigate the effect of INHD on previously unresearched outcome measures, using gold standard techniques including CMR, accelerometry and bioimpedance spectroscopy (BIS), to observe left ventricular (LV) structure and function, activity levels and body composition, respectively.

\section{METHODS AND ANALYSIS}

\section{Interventions to be measured}

This study will compare the effects of a 6-month programme of INHD on a number of outcome measures to matched control patients undergoing conventional daytime HD. This is a non-randomised controlled, feasibility study and patients in the intervention group can electively choose to switch to INHD.

The intervention group will undertake extended, INHD starting with a minimum of 5 hours and then building up incrementally, aiming for 7-8 hours three times per week according to their preference. The INHD programme will be delivered by three HD units within the East Midlands Renal Network, with patients arriving for their treatments in the evening and undergoing HD overnight.

The control group will receive CHD, defined as 4-hour HD sessions three times per week provided to patients during the day time.

As this trial is a feasibility study, no single measure has been selected as a primary outcome at this stage. A number of relevant variables have been identified that may be suitable as a primary outcome measure for a future multicentre randomised controlled trial. Collecting these data in our sample will inform the SD by which future sample size can be calculated.

\section{Patient recruitment}

The INHD intervention arm of the study will be a convenience sample of patients who have elected to start INHD. All patients electing to start INHD will be screened and all eligible patients will be approached to participate in the study.

In comparison, purposive sampling will be used to recruit patients to the control arm of the study. This is to ensure that the control group is comparable and well matched to the intervention group. The control group will be matched for age, gender, diabetes status and dialysis vintage.

To be eligible for the study patients must be prevalent adults on HD who have the capacity for consent and are able to undergo CMR. Further descriptions of inclusion and exclusion criteria for this study are shown in table 1 . Patients will be recruited to the intervention arm from three centres in the Leicester Renal Network providing INHD programmes. Control patients may be recruited from any centre in the Leicester Renal Network.

\section{Study timeline}

The study timeline is shown in figure 1. Patients in the intervention arm will be approached for consent 12 weeks after starting INHD. Initial assessments will include: baseline CMR (to be completed within 4 weeks 
Table 1 Inclusion and exclusion criteria for screening patient eligibility prior to recruitment into the MIDNIGHT study

\begin{tabular}{ll}
\hline Inclusion criteria & Exclusion criteria \\
\hline $\begin{array}{l}\text { On haemodialysis of } \\
\text { more than } 3 \text { months }\end{array}$ & $\begin{array}{l}\text { Unable to undergo CMR (due } \\
\text { to metal implants/prostheses, } \\
\text { claustrophobia or any other } \\
\text { reason) }\end{array}$ \\
$\begin{array}{l}\text { At least } 18 \text { years old } \\
\text { Have capacity to give } \\
\text { informed consent }\end{array}$ \\
$\begin{array}{l}\text { CMR, cardiac MRI; MIDNIGHT, Maintenance In-centre nocturnal } \\
\text { haemoDialysis: a feasibility study iNvestlGating the effects on } \\
\text { cardiac structure and pHysical funcTion. }\end{array}$
\end{tabular}

of the patient beginning INHD); physical performance assessments; body composition analysis; questionnaires exploring quality of life functional capacity, sleep quality, fatigue and symptom burden and blood sample collection. Patients will also be given an accelerometer to wear for 1-week for objective measurement of physical activity.

Interim assessments will take place after 3 months. This will involve repeating the questionnaires, body composition, physical performance and blood sampling. The interim assessments will be conducted over two visits, with the physical performance assessments completed on a separate day to blood sample collection to ensure that the blood sample is not affected by physical exertion involved in the performance tests.

Six months after baseline CMR all baseline assessments will be repeated at which point the protocol will be complete.

\section{Cardiovascular endpoints}

Cardiovascular disease in patients on HD is primarily driven by non-traditional risk factors, including chronic inflammation, chronic uraemia, myocardial stunning, vascular calcification and stiffening and endothelial damage. It has been documented that $75 \%$ of patients on HD have LV hypertrophy which is strongly linked to outcome and mortality in patients on HD. Previous studies using echocardiography suggest that INHD may reduce $L V$ mass by as much as $14 \mathrm{~g}$ in 6 months, and a recent study using a 1.5 Tesla $(1.5 \mathrm{~T})$ CMR showed that INHD led to a reduction of $8 \mathrm{~g}$ of $\mathrm{LV}$ mass in 1 -year. ${ }^{22}$

The CMR scan protocol timeline is shown in figure 2. We aim to conduct CMRs on a non-dialysis day within 24 hours of the previous HD session. Cardiac structure and function will be assessed using a $3 \mathrm{~T}$ CMR platform (Skyra, Siemens Medical Imaging, Erlangen, Germany). The CMR protocol will be similar to that previously described but without contrast administration. ${ }^{23}$ LV volumes and mass will be quantified with epicardial and endocardial contours of a contiguous stack of multiphase ventricular short axis cines (10-12 slices, $8 \mathrm{~mm}$ slice thickness, $25 \%$ gap) at end-diastole and end-systole. Native T1 and T2 mapping at mid-ventricular level will

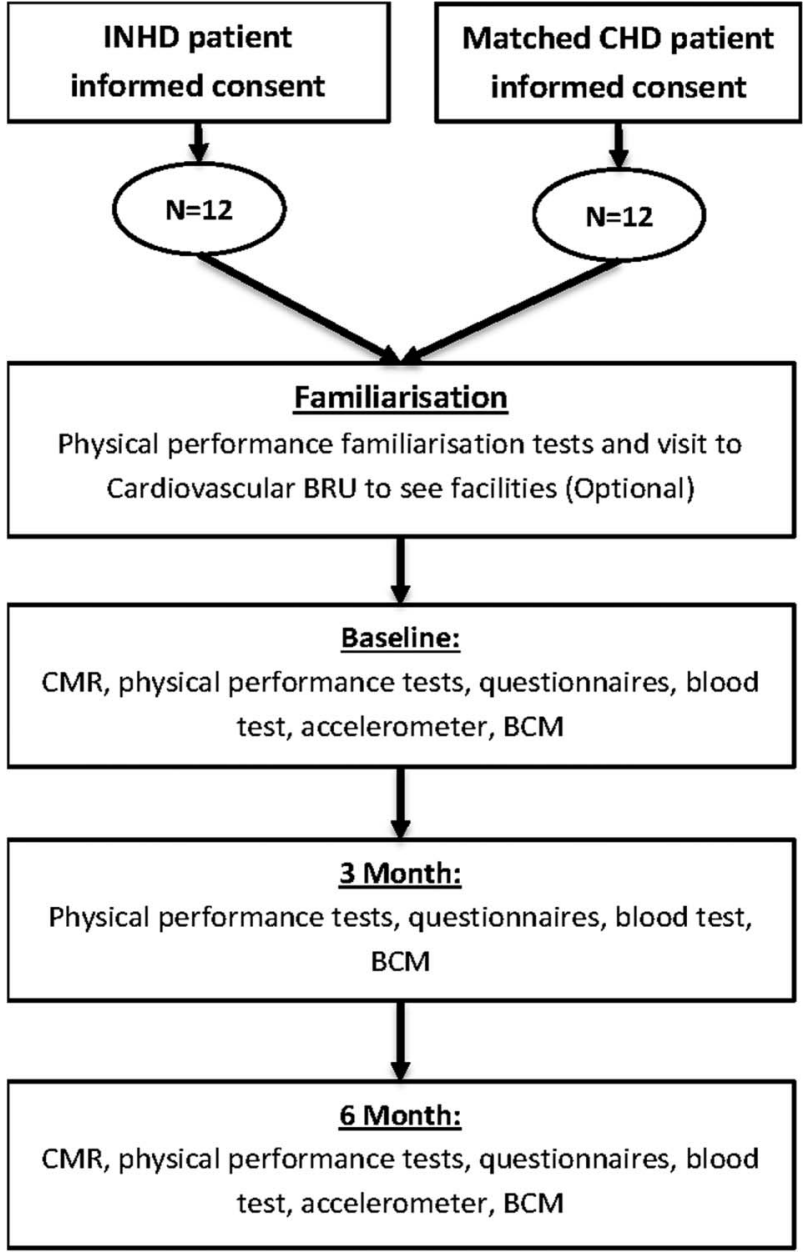

Figure 1 Flow chart showing when outcome measures will be collected. BCM, Body Composition Monitor; BRU, Biomedical Research Unit; CHD, conventional haemodialysis; CMR, cardiac MRI; INHD, in-centre nocturnal haemodialysis.

be undertaken for tissue characterisation, with noncontrast T1 mapping offering the opportunity to assess myocardial fibrosis. ${ }^{23} 24$ Tissue tracking will be used to assess systolic strain and diastolic strain. ${ }^{25}$ Arterial stiffness will be assessed by measuring the distensibility of the ascending and descending thoracic aorta. Changes in the cross-sectional luminal area of aorta in a $5 \mathrm{~mm}$ thick slice will be measured and concomitant measurement of blood pressure at the time of sequence acquisition will allow calculation of aortic distensibilty. ${ }^{26}$ We will also use CMR to measure aortic pulse wave velocity as a further measure of arterial stiffness.

\section{CMR image analysis}

All scans will be anonymised and analysed off-line. Both visual and quantitative analysis of CMR images will be undertaken, as per international guidelines. ${ }^{27}$ Quantitative analysis will be performed by a single operator using Food and Drug Administration (FDA) approved commercially available software. These will include: LV mass (g); enddiastolic volume $(\mathrm{mL})$; end-systolic volume $(\mathrm{mL})$; stroke 

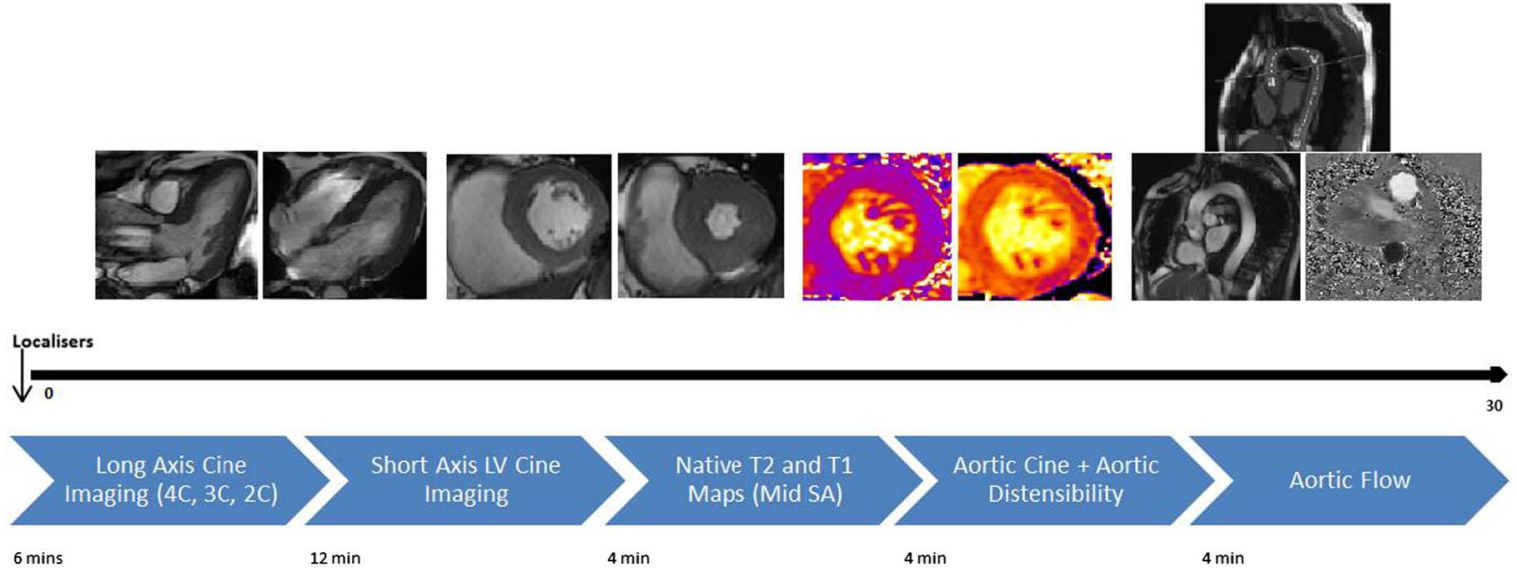

Figure 2 Protocol used for CMR procedures and pictures showing the images gained at those points. CMR, cardiac MRI; SA, short axis; LV, left ventricular.

volume $(\mathrm{mL})$; and ejection fraction $(\%)$. All volumetric data will be indexed to body surface area.

Calculation of $\mathrm{T} 1$ and $\mathrm{T} 2$ values will involve the drawing of epicardial and endocardial borders from a mid-short axis slice of the $\mathrm{LV}^{28} \mathrm{LV}$ strain and strain rate will be assessed as previously described. ${ }^{25}$ Manual drawing and propagation of endocardial and epicardial contours onto the end-diastolic image, will allow the calculation of LV endocardial and epicardial circumferential and longitudinal strain and strain rates.

Aortic distensibility will be analysed as previously described.$^{29}$ A region of interest using JIM V.6 (Xinapse software, UK) will be used to manually identify the ascending thoracic aortic area, which will be represented graphically against time. The following, validated formula will be used to determine aortic distensibility:

(maximum aortic area-minimum aortic area)/(minimum aortic area $\times \Delta P)$.

$\Delta \mathrm{P}$ is the brachial pulse pressure reading performed during CMR. ${ }^{30}$

\section{Physical performance}

Patients on HD have been shown to have reduced physical performance compared with healthy age-matched controls. ${ }^{31-34}$ This impacts on the ability to carry out activities of daily living, and it has also been shown that poor physical performance is linked to higher mortality risk. ${ }^{35}$ INHD will give patients more free time in the daytime to be physically active and engage in exercise activities, which may improve their physical performance.

We will measure physical performance using the short physical performance battery (SPPB) and the sit-to-stand 60 test (STS60). Both the SPPB and STS60 have been used by many research groups investigating the impact of exercise interventions in patients on HD. The SPPB comprises three simple tests: a balance test, requiring patients to stand in three increasingly difficult positions for $10 \mathrm{~s}$; a gait speed test, where patients are timed walking along a $4 \mathrm{~m}$ course; and a STS5, where patients stand up and sit down in a chair as quickly as possible five times, providing a measure of muscle power. ${ }^{37}$ The STS60 provides a surrogate marker of muscular endurance.

\section{Physical activity}

Owing to the scheduling and side effects of CHD, many patients on HD have a very sedentary lifestyle with extremely low activity levels. ${ }^{38-40}$ INHD will allow patients to be more active during the day.

Physical activity will be objectively measured using SenseWear Activity Monitors, which will be worn by the patients for 1-week. Measurements made by the accelerometer will provide values for average Metabolic Equivalent of Tasks (METs; a measure related to oxygen consumption that quantifies functional capacity $)^{41}$ for each patient during the time worn, energy expenditure and number of steps.

\section{Nutrition, body composition and blood biochemistry}

Current evidence suggests that INHD may improve nutritional aspects of patient's health, and may also increase body weight. ${ }^{15} 194243$ An increase in bodyweight is a desirable outcome for undernourished patients on HD, if the weight gained is lean tissue. ${ }^{44-46}$ However, the Frequent Haemodialysis Network (FHN) randomised-controlled trial that looked at nocturnal HD conducted on 5-7 days of the week at home, failed to show an improvement in body composition. ${ }^{47}$ This may have been due to the difference in their dialysis prescription, but may also be due to their assessment of body composition using single frequency bioimpedance analysis, a less-sensitive technique with disputable validity in patients on HD.

In the MIDNIGHT study, body composition will be analysed by BIS using a Body Composition Monitor (BCM; Fresenius, Bad Homburg, Germany). The BCM has been validated against a number of gold standard 
measurements for use in patients on HD. ${ }^{48}$ The calculations used by the BCM permit the accurate assessment of overhydration status in patients, as validated against expert clinical assessment. ${ }^{49}{ }^{50}$ Outcomes acquired include 'normally hydrated' lean tissue mass, 'normally hydrated' adipose tissue mass, intracellular fluid, extra cellular fluid, fat mass and body cell mass.

Data relating to clinical blood biochemistry and haematology will also be collected from routine monthly blood tests conducted as part of patient's standard care.

\section{Quality of life}

Previous research has shown that INHD may lead to significant improvements in patient's quality of life. ${ }^{51}$

We will assess changes in quality of life using the Short Form-12 questionnaire (SF-12; Quality Metrics), which has been previously validated for use in the renal disease population. ${ }^{52} 53$

\section{Symptom burden}

Longer dialysis is known to improve the clearance of small and middle weight molecules. ${ }^{54-56}$ The physical and psychological improvements previously documen$\operatorname{ted}^{51}$ are likely to be associated with a reduction in symptoms experienced by patients; however, previous studies have failed to report this. We will measure symptom burden using the Palliative Outcome Scale-Symptoms Renal (POS-S Renal) questionnaire. This 17-item questionnaire is specifically developed for use on patients with renal disease. ${ }^{57} 58$

We will also assess the levels of fatigue in these patients using the Functional Assessment of Chronic Illness Therapy—Fatigue (FACIT-F). ${ }^{59-61}$

\section{Sleep quality}

Patients on HD often experience disturbed and poor quality sleep. ${ }^{62}{ }^{63}$ Some data suggest that extended HD can improve sleep quality and reduce symptoms of sleep apnoea. ${ }^{64-66}$ The Pittsburgh Sleep Quality Index (PSQI) will be used to assess the impact of our INHD programme on patient's sleep quality. ${ }^{67-69}$

\section{Data analysis plan}

Demographic data on patients will be gathered and suitable comparisons (eg, $\chi^{2}$ test) will be made to ensure that the groups are well matched. All outcome measures will undergo appropriate omnibus tests to investigate statistical differences between patients and within patients. In addition to this we will look at associations between outcome measures and investigate potential interactions between variables, to generate hypotheses for future studies.

\section{ETHICS AND DISSEMINATION}

\section{Ethical and safety considerations}

The MIDNIGHT study protocol was reviewed by the Northampton Research and Ethics Committee (REC). This committee appraised the ethical implications of procedures that would be carried out during the study and the potential impact on patient rights and wellbeing. Favourable opinion from the REC means that due care and consideration has been made to preserve patient rights and the methodology is ethically justified; favourable opinion was provided on the 9 July 2015 (REC reference: 15/EM/0268). Further to this, local research and innovation approval was provided by University Hospitals of Leicester on 13 July 2015 (UHL11434).

Informed consent will be obtained from participants in line with recommendations set out in E6(R1) of the International Council for Harmonisation of Technical Requirements for Pharmaceuticals for Human Use (ICH) Good Clinical Practice guidelines.

\section{Dissemination plan}

We plan to present all data at national and international conferences. Furthermore, we will also publish the findings in international journals. We expect to publish our initial findings during late 2016/early 2017. Following the publication of our results participant level data will be available for collaborations, on request.

\section{Trial registration}

The MIDNIGHT study is registered with the ISRCTN (ISRCTN16672784). The registration process was initiated before the recruitment began; however, time constraints at one centre led to our first patient being recruited prior to payment clearing and completing our registration status. It is for this reason our registration status is listed as retrospective.

Twitter Follow Darren Churchward at @Darren_Churchy

Contributors DRC contributed to the drafting, review and final preparation of the manuscript. MPMG-B contributed to the drafting and review of the manuscript. GPM, RP and WP contributed to the conceptual design of the study and review of the manuscript. JOB contributed to the conceptual design of the study, review of the manuscript and gave final approval.

Funding This study was funded through a grant from the van Geest Heart and Cardiovascular Health Research Fund.

Disclaimer The views expressed in this publication are those of the authors and not necessarily those of the National Health Service, the sponsor or the funders.

Competing interests None declared.

Ethics approval NREC East Midlands-Northampton.

Provenance and peer review Not commissioned; externally peer reviewed.

Data sharing statement Anonymised participant level data will be made available to interested collaborators on request, following publication of final results manuscript.

Open Access This is an Open Access article distributed in accordance with the Creative Commons Attribution Non Commercial (CC BY-NC 4.0) license, which permits others to distribute, remix, adapt, build upon this work non- 
commercially, and license their derivative works on different terms, provided the original work is properly cited and the use is non-commercial. See: http:// creativecommons.org/licenses/by-nc/4.0/

\section{REFERENCES}

1. Gilg J, Pruthi R, Fogarty D. UK Renal Registry 17th Annual Report: chapter 1 UK renal replacement therapy incidence in 2013: national and centre-specific analyses. Nephron Physiol 2015;129(Suppl 1):1-29.

2. Rao A, Casula A, Castledine C. UK Renal Registry 17th Annual Report: chapter 2 UK renal replacement therapy prevalence in 2013 national and centre-specific analyses. Nephron Physiol 2015;129 (Suppl 1):31-56.

3. Stevens PE, O'donoghue DJ, de Lusignan S, et al. Chronic kidney disease management in the United Kingdom: NEOERICA project results. Kidney Int 2007;72:92-9.

4. Prescribing and primary care team health and social care information centre. Quality and outcomes framework achievement, prevalence and exceptions data 2012/13. 2013:1:5.

5. Gilg J, Caskey F, Fogarty D. UK Renal Registry 18th Annual Report chapter 1 UK renal replacement therapy incidence in 2014: national and centre-specific analyses. UK Renal Registry. 2016.

6. McIntyre CW, Burton JO, Selby NM, et al. Hemodialysis-induced cardiac dysfunction is associated with an acute reduction in global and segmental myocardial blood flow. Clin J Am Soc Nephrol 2008;3:19-26.

7. Burton JO, Jefferies HJ, Selby NM, et al. Hemodialysis-induced repetitive myocardial injury results in global and segmental reduction in systolic cardiac function. Clin J Am Soc Nephrol 2009;4:1925-31.

8. Burton JO, Korsheed S, Grundy BJ, et al. Hemodialysis-induced left ventricular dysfunction is associated with an increase in ventricular arrhythmias. Ren Fail 2008;30:701-9.

9. U.S. Renal Data System. USRDS 2006 Annual Data Report: Atlas of End-Stage Renal Disease in the United States. 2006

10. Abdel-Kader K, Unruh ML, Weisbord SD. Symptom burden, depression, and quality of life in chronic and end-stage kidney disease. Clin J Am Soc Nephrol 2009;4:1057-64.

11. Davison SN, Jhangri GS. Impact of pain and symptom burden on the health-related quality of life of hemodialysis patients. J Pain Symptom Manage 2010;39:477-85.

12. Cruz MC, Andrade C, Urrutia M, et al. Quality of life in patients with chronic kidney disease. Clinics (Sao Paulo) 2011;66:991-5.

13. Tennankore KK, Chan CT, Curran SP. Intensive home haemodialysis: benefits and barriers. Nat Rev Nephrol 2012;8:515-22.

14. Barraclough N, Mooney D, Mullins K, et al. Improved cardiac structure and function in a patient transitioned to in-centre nocturnal haemodialysis. Nephrology 2012;17:94.

15. Ok E, Duman S, Asci G, et al. Comparison of 4- and 8-h dialysis sessions in thrice-weekly in-centre haemodialysis: a prospective, case-controlled study. Nephrol Dial Transplant 2011;26:1287-96

16. Jin X, Rong S, Mei C, et al. Effects of thrice-weekly in-center nocturnal vs. conventional hemodialysis on integrated backscatter of myocardial tissue. Hemodial Int 2011;15:200-10.

17. Maduell F, Arias M, Durán CE, et al. Nocturnal, every-other-day, online haemodiafiltration: an effective therapeutic alternative. Nephrol Dial Transplant 2012;27:1619-31.

18. Lacson EJr, Wang W, Lester $\mathrm{K}$, et al. Outcomes associated with in-center nocturnal hemodialysis from a large multicenter program. Clin J Am Soc Nephrol 2010;5:220-6.

19. David $S$, Küempers $P$, Eisenbach GM, et al. Prospective evaluation of an in-centre conversion from conventional haemodialysis to an intensified nocturnal strategy. Nephrol Dial Transplant 2009;24:2232-40.

20. Nesrallah GE, Lindsay RM, Cuerden MS, et al. Intensive hemodialysis associates with improved survival compared with conventional hemodialysis. J Am Soc Nephrol 2012;23:696-705

21. Rocco MV, Larive B, Eggers PW, et al. Baseline characteristics of participants in the Frequent Hemodialysis Network (FHN) daily and nocturnal trials. Am J Kidney Dis 2011;57:90-100.

22. Wald R, Yan AT, Perl J, et al. Regression of left ventricular mass following conversion from conventional hemodialysis to thrice weekly in-centre nocturnal hemodialysis. BMC Nephrol 2012;13:3.

23. Singh A, Ford I, Greenwood JP, et al. Rationale and design of the PRognostic Importance of Mlcrovascular Dysfunction in asymptomatic patients with Aortic Stenosis (PRIMID-AS): a multicentre observational study with blinded investigations. BMJ Open 2013;3:e004348
24. Bull S, White SK, Piechnik SK, et al. Human non-contrast T1 values and correlation with histology in diffuse fibrosis. Heart 2013;99:932-7.

25. Khan JN, Singh A, Nazir SA, et al. Comparison of cardiovascular magnetic resonance feature tracking and tagging for the assessment of left ventricular systolic strain in acute myocardial infarction. Eur J Radiol 2015;84:840-8.

26. Chue CD, Edwards NC, Ferro CJ, et al. Effects of age and chronic kidney disease on regional aortic distensibility: a cardiovascular magnetic resonance study. Int J Cardiol 2013;168:4249-54.

27. Hundley WG, Bluemke D, Bogaert JG, et al. Society for Cardiovascular Magnetic Resonance guidelines for reporting cardiovascular magnetic resonance examinations. J Cardiovasc Magn Reson 2009;11:5

28. Mahmod M, Piechnik SK, Levelt E, et al. Adenosine stress native T1 mapping in severe aortic stenosis: evidence for a role of the intravascular compartment on myocardial T1 values. J Cardiovasc Magn Reson 2014;16:1.

29. Maroules CD, Khera A, Ayers C, et al. Cardiovascular outcome associations among cardiovascular magnetic resonance measures of arterial stiffness: the Dallas Heart Study. J Cardiovasc Magn Reson 2014;16:33.

30. Bramwell JC, Hill A. Velocity of transmission of the pulse-wave: and elasticity of arteries. Lancet 1922;199:891-2.

31. Painter $P$, Johansen $K L$. Improving physical functioning: time to be a part of routine care. Am J Kidney Dis 2006;48:167-70.

32. Segura-Ortí E. Exercise in haemodyalisis patients: a literature systematic review. Nefrologia 2010;30:236-46.

33. Smart N, Steele M. Exercise training in haemodialysis patients: a systematic review and meta-analysis. Nephrology 2011;16:626-32.

34. Smart N, McFarlane J, Cornelissen V. The effect of exercise therapy on physical function, biochemistry and dialysis adequacy in haemodialysis patients: a systematic review and meta-analysis Open J Nephrol 2013;3:25-36.

35. Roshanravan B, Robinson-Cohen C, Patel KV, et al. Association between physical performance and all-cause mortality in CKD. J Am Soc Nephrol 2013;24:822-30.

36. Painter $\mathrm{P}$, Roshanravan $\mathrm{B}$. The association of physical activity and physical function with clinical outcomes in adults with chronic kidney disease. Curr Opin Nephrol Hypertens 2013;22:615-23.

37. Guralnik JM, Ferrucci L, Pieper CF, et al. Lower extremity function and subsequent disability: consistency across studies, predictive models, and value of gait speed alone compared with the short physical performance battery. J Gerontol A Biol Sci Med Sci 2000;55:M221-31.

38. Thorp AA, Owen N, Neuhaus M, et al. Sedentary behaviors and subsequent health outcomes in adults: a systematic review of longitudinal studies, 1996-2011. Am J Prev Med 2011;41:207-15.

39. Anderton N, Giri A, Wei G, et al. Sedentary behavior in individuals with diabetic chronic kidney disease and maintenance hemodialysis. $J$ Ren Nutr 2015;25:364-70.

40. Wilmot EG, Edwardson CL, Achana FA, et al. Sedentary time in adults and the association with diabetes, cardiovascular disease and death: systematic review and meta-analysis. Diabetologia 2012:55:2895-905.

41. Jetté $M$, Sidney K, Blümchen G. Metabolic equivalents (METS) in exercise testing, exercise prescription, and evaluation of functional capacity. Clin Cardiol 1990;13:555-65.

42. Powell JR, Oluwaseun O, Woo YM, et al. Ten years experience of in-center thrice weekly long overnight hemodialysis. Clin J Am Soc Nephrol 2009;4:1097-101.

43. Alloatti S, Molino A, Manes M, et al. Long nocturnal dialysis. Blood Purif 2002:20:525-30

44. Kopple JD, Zhu X, Lew NL, et al. Body weight-for-height relationships predict mortality in maintenance hemodialysis patients. Kidney Int 1999;56:1136-48.

45. Leavey SF, McCullough K, Hecking E, et al. Body mass index and mortality in 'healthier' as compared with 'sicker' haemodialysis patients: results from the Dialysis Outcomes and Practice Patterns Study (DOPPS). Nephrol Dial Transplant 2001;16:2386-94.

46. Fleischmann E, Teal N, Dudley J, et al. Influence of excess weight on mortality and hospital stay in 1346 hemodialysis patients. Kidney Int 1999:55:1560-7.

47. Kaysen GA, Greene T, Larive B, et al. The effect of frequent hemodialysis on nutrition and body composition: Frequent Hemodialysis Network Trial. Kidney Int 2012;82:90-9.

48. Wabel P, Chamney P, Moissl U, et al. Importance of whole-body bioimpedance spectroscopy for the management of fluid balance. Blood Purif 2009;27:75-80. 
49. Wabel P, Rode C, Moissl U, et al. Accuracy of bioimpedance spectroscopy (BIS) to detect fluid status changes in hemodialysis patients. Nephrol Dial Transplant 2007;22:VI129.

50. Chamney PW, Wabel P, MoissI UM, et al. A whole-body model to distinguish excess fluid from the hydration of major body tissues. Am $J$ Clin Nutr 2007;85:80-9.

51. Graham-Brown MP, Churchward DR, Smith AC, et al. A 4-month programme of in-centre nocturnal haemodialysis was associated with improvements in patient outcomes. Clin Kidney J 2015;8: sfv096.

52. Lacson EJr, Xu J, Lin SF, et al. A comparison of SF-36 and SF-12 composite scores and subsequent hospitalization and mortality risks in long-term dialysis patients. Clin J Am Soc Nephrol 2010;5:252-60.

53. Singh A, Gnanalingham K, Casey A, et al. Quality of life assessment using the Short Form-12 (SF-12) questionnaire in patients with cervical spondylotic myelopathy: comparison with SF-36. Spine (Phila Pa 1976) 2006;31:639-43.

54. Suri R, Depner TA, Blake PG, et al. Adequacy of quotidian hemodialysis. Am J Kidney Dis 2003;42(Suppl 1):42-8.

55. Raj DS, Ouwendyk M, Francoeur R, et al. Beta(2)-microglobulin kinetics in nocturnal haemodialysis. Nephrol Dial Transplant 2000;15:58-64.

56. Chertow GM, Levin NW, Beck GJ, et al. FHN Trial Group. In-center hemodialysis six times per week versus three times per week. N Engl J Med 2010;363:2287-300.

57. Saini T, Murtagh FE, Dupont PJ, et al. Comparative pilot study of symptoms and quality of life in cancer patients and patients with end stage renal disease. Palliat Med 2006;20:631-6.

58. Bausewein C, Le Grice C, Simon S, et al. The use of two common palliative outcome measures in clinical care and research: a systematic review of POS and STAS. Palliat Med 2011;25:304-13.
59. Chandran V, Bhella S, Schentag C, et al. Functional Assessment of Chronic Illness Therapy-Fatigue scale is valid in patients with psoriatic arthritis. Ann Rheum Dis 2007;66:936-9.

60. Jhamb M, Weisbord SD, Steel JL, et al. Fatigue in patients receiving maintenance dialysis: a review of definitions, measures, and contributing factors. Am J Kidney Dis 2008;52:353-65.

61. Cella DF, Tulsky DS, Gray G, et al. The functional assessment of cancer therapy scale: development and validation of the general measure. J Clin Oncol 1993;11:570-9.

62. Iliescu EA, Yeates KE, Holland DC. Quality of sleep in patients with chronic kidney disease. Nephrol Dial Transplant 2004;19:95-9.

63. Mavanur M, Sanders M, Unruh M. Sleep disordered breathing in patients with chronic kidney disease. Indian J Med Res 2010;131:277-84.

64. Koch BCP, van der Putten K, Van Someren EJW, et al. Impairment of endogenous melatonin rhythm is related to the degree of chronic kidney disease (CREAM study). Nephrology Dialysis Transplantation 2010;25:513-19.

65. Koch BC, Hagen EC, Nagtegaal JE, et al. Effects of nocturnal hemodialysis on melatonin rhythm and sleep-wake behavior: an uncontrolled trial. Am J Kidney Dis 2009;53:658-64.

66. Beecroft JM, Hoffstein V, Pierratos A, et al. Nocturnal haemodialysis increases pharyngeal size in patients with sleep apnoea and end-stage renal disease. Nephrol Dial Transplant 2008;23:673-9.

67. Carpenter JS, Andrykowski MA. Psychometric evaluation of the Pittsburgh Sleep Quality Index. J Psychosom Res 1998;45:5-13.

68. Beck SL, Schwartz AL, Towsley G, et al. Psychometric evaluation of the Pittsburgh Sleep Quality Index in cancer patients. J Pain Symptom Manage 2004;27:140-8.

69. Buysse DJ, Reynolds CF, Monk TH, et al. The Pittsburgh Sleep Quality Index: a new instrument for psychiatric practice and research. Psychiatry Res 1989;28:193-213. 